\section{Monoklonale Anti-} körper - eine neue Therapiemodalität zur Behandlung des malignen NonHodgkin-Lymphoms

\author{
U. Breitenstein
}

morphologischen Kriterien auch charakteristische immunphänotypische und zytogenetische Veränderungen einzelner Lymphomsubtypen [1]. Die Einführung des "International Prognostic Index" erlaubt durch einfache Kriterien eine bessere prognostische Einschätzung der Patienten mit diffusen grosszelligen B-Zellen-NHL [2]. Der prognostische Index kann auf niedrig maligne NHL vom follikulären Typ übertragen werden, wo er ebenfalls eine klare Abstufung von prognostischen Subgruppen ermöglicht. Auf der therapeutischen Ebene musste leider bei den grosszelligen diffusen NHL (DLCL) festgestellt werden, dass die während der 80er Jahre entwickelten 3. Generationstherapien der Standardtherapie mit Endoxan, Doxorubicin, Vincristin und Prednison (CHOP) nicht überlegen sind [3]. Leider zeigte sich auch, dass für Hochrisikopatienten mit DLCL die Hochdosistherapie mit autologer Stammzelltransplantation in 1. Remission keine Verbesserung des Überlebens bewirkt $[4,5]$. Lediglich eine randomisierte Studie mit einem sequentiellen Hochdosisschema zeigt grenzwertig bessere Resultate in bezug auf das rezidivfreie Überleben [6]. Klarer ist der Stellenwert der Hochdosistherapie mit autologer Stammzelltransplantation im Falle eines Rezidivs des DLCL [7]. Hier ist das bessere kurative Potential bei nicht lokalisierten Rezidivstadien (>Stadium I) belegt und die Indikation zur autologen Dosisintensivierung ist klar gegeben. Bei den follikulären NHL entfachte die Einführung der neuen Purinantagonisten (Fludarabin, 2-CDA, Pentostatin) neue Hoffnungen. Diese Medikamente bewirken deutlich höhere Ansprechraten und können auch bei stark vorbehandelten Patienten gute Wirkung entfalten. Leider hat sich gezeigt, dass trotz der Einführung dieser Medikamentengruppe die Prognose der Patienten mit niedrig malignen NHL in den letzten Jahrzehnten nicht verbessert werden konnte. Auch Interferon-Alpha als konkommittierende Behandlung oder als Maintenance-Therapie hat sich bei diesem Lymphomtyp nicht durchsetzen können. Der Stellenwert der Hochdosistherapie bei follikulären Lymphomen ist nicht etabliert und aktuell Gegenstand von klinischen Studien. Bei der neu definierten Entität des Mantelzell-Lymphoms (MZL) sind keine wesentlichen therapeutischen Durchbrüche gelungen. Diese Form hat weiterhin die schlechteste Prognose aller NHL und die Erkrankung ist häufig therapieresistent.

Insgesamt scheint die zytostatische Therapie am Ende des Jahrtausends in einer Sackgasse zu stehen und die Einführung neuer therapeutischer monoklonaler Antikörper birgt die Hoffnung, dass weitere Fortschritte in der Behandlung vieler Lymphomtypen erreicht werden können.
Korrespondenz:

Dr. med. Urs Breitenstein

Onkozentrum

Klinik Im Park

Kappelistrasse 35

CH-8002 Zürich

\section{Monoklonale Antikörper}

Bei therapeutischen monoklonalen Antikörpern (Mab) handelt es sich um gentechnologisch hergestellte Immunglobuline der Klasse IgG, welche über die hypervariable Aminosäurensequenz an ein tumor- 
spezifisches Antigen binden können und über ihre konstante Region (Fc-Domäne) die Kapazität haben, eine Antikörper-vermittelte zelluläre Zytotoxizität (ADCC) und eine komplementabhängige Zytotoxizität (CDCC) zu bewirken. Die Antikörper werden über Myelomzellkulturen hergestellt, welche durch eine Hybridisierung einer spezifisch immunisierten B-Zelle und einer immortalen Myelomzelle entstanden sind. Durch zusätzliche molekularbiotechnische Veränderungen entstehen aus monoklonalen murinen Antikörpern chimäre monoklonale Antikörper (gemischt murin/human, hypervariabler Region und Teile der Leichtketten tierischen Ursprungs) oder $h u$ manisierte monoklonale Antikörper, bei denen lediglich die hypervariable Aminosäurensequenz murinen Ursprungs ist und alle übrigen Antikörperanteile aus humanen Sequenzen bestehen. Durch diese Veränderung der Antikörper kann die Zytotoxizität des Antikörpers erhöht werden, da murine Antikörper häufig eine limitierte Fähigkeit haben, humane immunologische Effektorzellen oder humanes Komplement $\mathrm{zu}$ aktivieren. Humanes IgG 1 oder murines IgG 2 bzw. murines IgG 3 sind die geeignetsten IgG-Subtypen, um das humane Komplementsystem auszulösen. Die humanen Antikörpersubtypen IgG 1 und $\operatorname{IgG} 3$ vermitteln am effektivsten eine ADCC. Die Chimärisierung oder Humanisierung der Antikörperstruktur bewirkt zudem eine verminderte Immunogenität des Antikörpers. Neutralisierende humane Anti-MausAntikörper (HAMA) werden zu 25-50\% gebildet und führen $\mathrm{zu}$ einem raschen Wirkungsverlust des Antikörpers. Durch die partielle Humanisierung der Antikörperstruktur kann eine Antikörperbildung fast vollständig verhindert werden. Somit konnte eine wichtige Hürde in der Entwicklung der Mab überwunden werden. Ein weiteres Problem stellt die Kreuzreaktion der gegen Tumorantigene gerichteten Antikörper mit Antigenen von Zellen des Normalgewebes dar. Dies kann einerseits zu verminderter Aktivität des Mab an der malignen Zielzelle und andererseits zu potentiellen Organtoxizitäten führen. Zusätzlich kann die Wirkung eines Mab durch die Modulation durch "shedding" und durch die Internalisation des oberflächlichen Zielantigens verändert bzw. behindert werden. Durch die Selektion von stabilen Zielantigenen wird diese Problematik umgangen. In der Behandlung des malignen NHL haben sich die Oberflächenantigene CD20 und CD52 als geeignetste Zielantigene (Target) erwiesen. Als idealer Angriffspunkt für eine Immuntherapie präsentiert sich das CD20-Antigen. Es handelt sich dabei um ein 35 kD schweres, membranständiges Phosphoprotein, welches nur auf B-Vorläuferzellen sowie reifen BZellen exprimiert wird. Bei Ausreifung der B-Zelle zur antikörperproduzierenden Plasmazelle kommt es zum Verlust der CD20-Expression. Knochenmarkstammzellen werden durch eine Anti-CD20-Antikörpertherapie nicht beeinflusst, da sie ebenfalls keine Expression für dieses Oberflächenantigen aufweisen. Daraus resultiert eine praktisch fehlende Hämatotoxizität dieses Mab. Zusätzlich handelt es sich beim CD20Antigen um ein stabiles Membranprotein, das an der
Zelloberfläche nicht moduliert oder internalisiert werden kann. Das Antigen zirkuliert im Plasma nicht als freies Antigen und blockiert daher den Antikörper nicht vor Erreichen der malignen B-Zelle. Als erster therapeutischer Anti-CD20-Antikörper wurde der Maus/Mensch-chimäre monoklonale Antikörper Rituximab (IDEC-C2B8; Mabthera ${ }^{\circledR}$, Roche) entwickelt und für den therapeutischen Einsatz freigegeben. Er besteht in seiner variablen Region (CD20Bindungsstelle) aus einem murinen IgG 1 und in der konstanten Region aus einem humanen IgG 1 Kappa. Die Bindung des Antikörpers an nicht-neoplastische B-Zellen führt nicht zur Entwicklung eines B-zellulären Immundefektes. Es können sich leicht verminderte IgG- und IgM-Serumspiegel nach Behandlung mit Rituximab entwickeln. Eine vermehrte Infektionsrate oder Auftreten von opportunistischen Infekten ist nach Einsatz dieses Antikörpers nicht beschrieben. Die Inzidenz von neutralisierenden antichimären Antikörpern ist gering $(<1 \%)$. Die empfohlene Dosis beträgt $375 \mathrm{mg} / \mathrm{m}^{2}$ iv, wöchentlich verabreicht für 4 Dosierungen. Eine längere Therapiedauer über 8 Wochen verbessert die Ansprechrate und die Remissionsdauer nicht [8].

Mehr als 90\% aller B-Zellen NHL exprimieren CD20 und eröffnen damit ein breites Spektrum verschiedenster Typen von B-Zellen NHL, welche sich für eine Anti-CD20-Antikörpertherapie eignen.

\section{Follikuläres Non-Hodgkin Lymphom}

In einer multizentrischen Studie (Pivotal-Trial) wurden 166 Patienten mit niedrig malignen oder follikulären NHL im Rezidiv oder nach Versagen einer Primärtherapie mit Rituximab $375 \mathrm{mg} / \mathrm{m}^{2}$ wöchentlich für 4 Wochen behandelt [9]. Die Resultate waren vielversprechend. Ein äusserst hoher Anteil entwickelte eine quantifizierbare Tumorverkleinerung. $48 \%$ erreichten eine partielle oder komplette Remission. Nur der kleinste Teil der Patienten zeigte eine Tumorprogression unter der Antikörperbehandlung. Die Ansprechrate stand im Abhängigkeit der Anzahl vorangehender Rezidive. Nach einem 1. Rezidiv lag die Ansprechrate bei 57\%, nach 2. Rezidiv bei 46\% und nach 3. Rezidiv bei 38\%. Die Subgruppenanalyse ergab lediglich statistisch signifikante Unterschiede in der Ansprechrate in Abhängigkeit vom Lymphomtyp (IWF A 12\% versus IWF B-D 58\%), bei Knochenmarksbefall (40\% ohne versus 59\% mit) und in Abhängigkeit, ob vorgehend eine autologe Knochenmark- oder periphere Stammzelltransplantation durchgeführt wurde. Das Alter, die Tumorgrösse und der LDH-Wert sowie das Ausmass der Vorbehandlung bzw. eine Chemotherapieresistenz beeinflussten die Ansprechrate nicht. Die mediane Zeit bis zur Tumorprogression lag bei den Respondern bei 13,0 Monaten. In dieser Studie konnte ebenfalls ein günstiges Nebenwirkungsprofil aufgezeigt werden. Die Häufigkeit und der Schweregrad der Nebenwirkungen nahmen im Verlaufe der 4 Infusionsbehandlungen deutlich ab (1. Infusion 73\%, 4. Infusion 20\%). Dass die 
Lymphommasse die Ansprechrate nicht beeinflusst, konnten Davis et al. bestätigen [10]. In dieser Studie wurden 31 Patienten mit rezidiviertem oder therapierefraktärem niedrig malignem oder follikulärem NHL mit Tumormanifestation $>10 \mathrm{~cm}$ ("bulky disease») mit einer Rituximab-Standarddosis behandelt, und es konnte ein Ansprechen von 43\% erreicht werden. Eine nochmalige Behandlung mit dem CD20-Antikörper bei bereits durchgeführter Rituximabtherapie war Gegenstand einer kürzlich publizierten Arbeit [11]. Alle Patienten waren mit Rituximab in einem medianen Intervall von 14,5 Monaten vorbehandelt. 40\% erreichten wiederum ein Ansprechen, wobei die komplette Remissionsrate bei 11\% und die partielle Remissionsrate bei 30\% lag. In keinem der Fälle waren nach Vorbehandlungen antichimäre Antikörper nachweisbar. Die mediane Zeit bis zur Tumorprogression lag bei 17,8 Monaten (5,4-26,6 Monate). Durch die Kombinationsbehandlung von Rituximab mit der anthrazyklinhaltigen Standardchemotherapie CHOP konnte bei 40 Patienten mit niedrig malignem oder follikulärem NHL gezeigt werden, dass sehr hohe Ansprechraten von 95\% (komplette Remission 55\%, Partialremission 40\%) erreicht werden können [12].

\section{Mantelzell-Lymphom}

Das MZL stellt aus chemotherapeutischer Sicht ein Problem dar, da ein grosser Teil dieser Patienten an einer therapieresistenten Erkrankung leiden. Die bis anhin vorliegenden Daten für Rituximab sind ebenfalls vielversprechend [13]. Sowohl bei neu diagnostizierten Fällen als auch bei chemotherapeutisch vorbehandelten Patienten konnten äquivalente Ansprechraten von 38\% (neu diagnostizierte Fälle) bzw. 34\% (vorbehandelte MZL) erreicht werden. Die komplette Remissionsrate lag in beiden Gruppen um die 15\%. Die Ansprechraten beim follikulären NHL und beim MZL konnten durch die schweizerische Phase2-Studie der SAKK im wesentlichen bestätigt werden [14]. Die Ansprechrate beim follikulären NHL lag bei 52\%. Beim MZL liegt die Ansprechrate im Vergleich zur oben erwähnten Studien mit 22\% tiefer.

\section{Lymphozytisches NHL und Immunozytom}

Schon die Pivotal-Studie [9] zeigte, dass die Ansprechrate der verschiedenen niedrig malignen NHL sehr unterschiedlich ist. Die Ansprechrate bei Patienten mit lymphozytischem NHL (IWF A) liegt nur bei $12 \%$. Diese relativ niedrige Ansprechrate (14\%) konnte durch eine weitere Studie an einem kleinen Patientenkollektiv bestätigt werden [13]. Die Wirkung des Rituximab beim Immunozytom (Morbus Waldenström) liegt etwas höher (Ansprechrate 28\%, alles Partialremissionen). Auch chemotherapieresistente Patienten mit Immunozytom können auf die monoklonale Anti-CD20-Antikörpertherapie (3/7 Patienten) ansprechen [15]. Im Gegensatz zu den follikulären NHL zeigten sich bei den leukämischen «low
grade»-Lymphomen und bei chronisch lymphatischer Leukämie signifikant höhere Nebenwirkungsraten ab einer Lymphozytose $>50$ 000/ul [16]. Bei diesen Patienten wird ein Zytokine-release-Syndrom beschrieben, welches noch unter Rituximabinfusion Schüttelfrost, Nausea/Erbrechen, Hypotonie und Dyspnoe verursacht. Laborchemisch kann es zu Anstieg von Leberenzymen und zur Aktivierung von Gerinnungsparametern kommen. Etwa 90 Minuten nach Infusionsbeginn können erhöhte TNF-alpha- und IL6Serumspiegel gemessen werden. Ähnliche Beobachtungen stellten Byrd et al. bei Patienten mit hoher peripherer Tumorzellzahl fest [17]. Letale Ausgänge wurden bereits in Einzelberichten beschrieben. Für dieses Patientenkollektiv mit einer Lymphozytose $>50$ 000/ul wird zur Vermeidung von schwereren Nebenwirkungen eine fraktionierte Dosisverabreichung der Rituximab-Standarddosierung von $375 \mathrm{mg} / \mathrm{m}^{2}$ empfohlen (Tag 1 absolut $50 \mathrm{mg}$, Tag 2 absolut 150 mg, Tag 3 Rest der Gesamtdosis).

\section{Diffuses grosszelliges B-Zellen-NHL (DLCL)}

Die Datenlage bezüglich des Einsatzes von Rituximab bei DLCL ist noch recht mager. In einer Phase-2-Studie mit kleinem gemischtem Kollektiv von 54 Patienten mit DLCL und MZL konnte in der Gruppe der DLCL eine Ansprechrate von 37\% erreicht werden [18].

\section{Posttransplantäre lymphoproliferative Erkrankungen}

In einer retrospektiven Zusammenstellung aus 15 französischen Transplantationszentren werden 32 Patienten mit überwiegend soliden Organtransplantationen mit posttransplantären B-lymphoproliferativen Erkrankungen beschrieben [19]. Rituximab wurden mehrheitlich als Erstlinientherapie nach Versagen auf eine Reduktion der immunsuppressiven Behandlung verabreicht. Die Ansprechrate liegt bei $79 \%$, wobei mehrheitlich komplette Remissionen erzielt wurden.

\section{Zusammenfassung}

Die Therapieresultate der monoklonalen Antikörpertherapie mit dem Anti-CD20-Antikörper Rituximab sind vielversprechend. Alleine oder in Kombination mit Chemotherapie sind Ansprechraten sehr hoch. Auch chemotherapieresistente Erkrankungen können auf diese immunologische Therapie ansprechen. Der Stellenwert dieser neuen Therapiemodalität muss jedoch noch genauer definiert werden. Rituximab als Konsolidationstherapie nach autologer Dosisintensivierung und zum "in-vivo purging" vor autologer Stammzellsammlung wird Gegenstand künftiger Studien sein. Ebenso vielversprechend sind die ersten Therapieresultate von konjugierten monoklonalen Antikörpern. Die limitierte antitumorale Wirkung von unkonjugierten Antikörpern kann durch Konju- 
gation mit einem Radionuklid $\left(\operatorname{Iod}^{131}\right.$, Ytrium $^{90}$ ) potenziert werden. Ebenso könnte die Wirkung eines Mab durch Konjugation mit einem Toxin oder einem zytotoxischen Medikament verbessert werden.

Es haben sich zu Beginn des neuen Jahrtausends vielversprechende Therapiewege zur Behandlung des malignen NHL eröffnet.

\section{Literatur}

1 Harris NL, Jaffe ES, Stein H, Banks PM, Chan JK, Cleary ML, et al. A revised European-American classification of lymphoid neoplasms: a proposal from the International Lymphoma Study Group. Blood 1994;84:1361-92.

2 A predictive model for aggressive non-Hodgkin's lymphoma. The International Non-Hodgkin's Lymphoma Prognostic Factors Project. N Engl J Med. 1993;329:987-94.

3 Fisher RI, Gaynor ER, Dahlberg S, Oken MM, Grogan TM, Mize EM, et al. Comparison of a standard regimen (CHOP) with three intensive chemotherapy regimens for advanced non-Hodgkin's lymphoma. N Engl J Med 1993;328:1002-6.

4 Haioun C, Lepage E, Gisselbrecht C, Coiffier B, Bosly A, Tilly H, et al. Comparison of autologous bone marrow transplantation with sequential chemotherapy for intermediategrade and high-grade non-Hodgkin's lymphoma in first complete remission: a study of 464 patients. Groupe d'Etude des Lymphomes de l'Adulte. J Clin Oncol 1994;12:2543-51.

5 Santini G, Salvagno L, Leoni P, Chisesi T, De Souza C, Sertoli MR, et al. VACOP-B versus VACOP-B plus autologous bone marrow transplantation for advanced diffuse nonHodgkin's lymphoma: results of a prospective randomized trial by the non-Hodgkin's Lymphoma Cooperative Study Group. J Clin Oncol 1998;16:2796-802.

6 Gianni AM, Bregni M, Siena S, Brambilla C, Di Nicola M, Lombardi F, et al. High-dose chemotherapy and autologous bone marrow transplantation compared with MACOP-B in aggressive B-cell lymphoma. N Engl J Med 1997;336:1290-7.

7 Philip T, Guglielmi C, Hagenbeek A, Somers R, Van der Lelie H, Bron D, et al. Autologous bone marrow transplantation as compared with salvage chemotherapy in relapses of chemotherapy-sensitive non-Hodgkin's lymphoma. N Engl J Med 1995;333:1540-5.

8 Piro LD, White CA, Grillo-Lopez AJ, Janakiraman N, Saven A, Beck TM, et al. Extended Rituximab (anti-CD20 monoclonal antibody) therapy for relapsed or refractory low-grade or follicular non-Hodgkin's lymphoma. Ann Oncol 1999;10: 655-61.

9 McLaughlin, Grillo-Lopez AJ, Link BK, Levy R, Czuczman MS, Williams ME, et al. Rituximab chimeric anti-CD20 monoclonal antibody therapy for relapsed indolent lymphoma: half of patients respond to a four-dose treatment program. J Clin Oncol 1998;16:2825-33.
10 Davis TA, White CA, Grillo-Lopez AJ, Velasquez WS, Link B, Maloney DG, et al. Single-agent monoclonal antibody efficacy in bulky non-Hodgkin's lymphoma: results of a phase II trial of rituximab. J Clin Oncol 1999;17:1851-7.

11 Davis TA, Grillo-Lopez AJ, White CA, McLaughlin P, Czuczman MS, Link BK, et al. Rituximab anti-CD20 monoclonal antibody therapy in non-Hodgkin's lymphoma: safety and efficacy of re-treatment. J Clin Oncol 2000;18:3135-43.

12 Czuczman MS, Grillo-Lopez AJ, White CA, Saleh M, Gordon L, LoBuglio AF, et al. Treatment of patients with lowgrade B-cell lymphoma with the combination of chimeric anti-CD20 monoclonal antibody and CHOP chemotherapy. J Clin Oncol 1999;17:268-76.

13 Foran JM, Rohatiner AZ, Cunningham D, Popescu RA, Solal-Celigny P, Ghielmini M, et al. European phase II study of rituximab (chimeric anti-CD20 monoclonal antibody) for patients with newly diagnosed mantle-cell lymphoma and previously treated mantle-cell lymphoma, immunocytoma, and small B-cell lymphocytic lymphoma. J Clin Oncol 2000; 18:317-24.

14 Ghielmini M, Schmitz SF, Burki K, Pichert G, Betticher DC, Stupp R, et al. The effect of Rituximab on patients with follicular and mantle-cell lymphoma. Swiss Group for Clinical Cancer Research (SAKK). Ann Oncol 2000;11(Suppl 1):123-6. 15 Byrd JC, White CA, Link B, Lucas MS, Velasquez WS, Rosenberg J, Grillo-Lopez AJ. Rituximab therapy in Waldenstrom's macroglobulinemia: preliminary evidence of clinical activity. Ann Oncol 1999;10:1525-7.

16 Winkler U, Jensen M, Manzke 0, Schulz H, Diehl V, Engert A. Cytokine-release syndrome in patients with B-cell chronic lymphocytic leukemia and high lymphocyte counts after treatment with an anti-CD20 monoclonal antibody (rituximab, IDEC-C2B8). Blood 1999;94:2217-24.

17 Byrd JC, Waselenko JK, Maneatis TJ, Murphy T, Ward FT, Monahan BP, et al. Rituximab therapy in hematologic malignancy patients with circulating blood tumor cells: association with increased infusion-related side effects and rapid blood tumor clearance. J Clin Oncol 1999;17:791-5.

18 Coiffier B, Haioun C, Ketterer N, Engert A, Tilly H, Ma D, et al. Rituximab (anti-CD20 monoclonal antibody) for the treatment of patients with relapsing or refractory aggressive lymphoma: a multicenter phase II study. Blood 1998;92: 1927-32.

19 Milpied N, Vasseur B, Parquet N, Garnier JL, Antoine C, Quartier P, et al. Humanized anti-CD20 monoclonal antibody (Rituximab) in post transplant B-lymphoproliferative disorder: a retrospective analysis on 32 patients. Ann Oncol 2000;11(Suppl 1):113-6. 